\title{
Maternal dietary patterns and risk of adverse pregnancy (hypertensive disorders of pregnancy and gestational diabetes mellitus) and birth (preterm birth and low birth weight) outcomes: a systematic review and meta-analysis
}

\author{
Kelemu Tilahun Kibret ${ }^{1, *}$, Catherine Chojenta ${ }^{1}$, Ellie Gresham ${ }^{2}$, Teketo K Tegegne ${ }^{1}$ \\ and Deborah Loxton ${ }^{1}$ \\ 'Priority Research Centre for Generational Health and Aging, School of Medicine and Public Health, University of \\ Newcastle, University Drive, Callaghan, NSW 2308, Australia: ${ }^{2}$ Health Intelligence Unit, Orange Health Service, \\ Western NSW Health, Orange, New South Wales, Australia
}

Submitted 15 March 2018: Final revision received 28 August 2018: Accepted 3 September 2018: First published online 15 October 2018

\begin{abstract}
Objective: Epidemiological studies have indicated that dietary patterns during pregnancy are associated with adverse pregnancy and birth outcomes such as hypertensive disorders of pregnancy (HDP), gestational diabetes mellitus (GDM), preterm birth (PTB) and low birth weight (LBW). However, the results of these studies are varied and inconsistent. The present study aimed to assess the association between dietary patterns and the risk of adverse pregnancy and birth outcomes.

Design: Systematic review and meta-analysis. Seven databases were searched for articles. Two reviewers performed the study selection and data extraction. A random-effects model was used to estimate pooled effect sizes of eligible studies. Setting: Studies conducted all over the world were incorporated.

Subjects: The review focused on pregnant women.

Results: A total of twenty-one studies were identified. Adherence to a healthy dietary pattern (intake of vegetables, fruits, legumes, whole grains) was significantly associated with lower odds (OR; 95\% CI) of pre-eclampsia ( 0.78 ; $\left.0.70,0.86 ; I^{2}=39 \cdot 0 \%, P=0.178\right), \operatorname{GDM}\left(0 \cdot 78 ; 0.56,0.99 ; I^{2}=68.6 \%, P=0.013\right)$ and PTB $\left(0 \cdot 75 ; 0.57,0.93 ; I^{2}=89 \cdot 6 \%, P=0.0001\right)$.

Conclusions: Our review suggests that dietary patterns with a higher intake of fruits, vegetables, legumes, whole grains and fish are associated with a decreased likelihood of adverse pregnancy and birth outcomes. Further research should be conducted in low-income countries to understand the impact of limited resources on dietary intake and adverse pregnancy and birth outcomes.
\end{abstract}

Keywords

Dietary patterns Dietary intake

Pregnancy

Pregnant women
Hypertensive disorders of pregnancy (HDP) are a group of conditions related to high blood pressure during pregnancy, proteinuria and in some cases convulsions ${ }^{(1)}$. HDP are responsible for increased morbidity and mortality in mothers and newborns, accounting for approximately $14 \%$ of maternal deaths globally between 2003 and $2009^{(2)}$. According to an analysis of international cohorts from six countries (Australia/New Zealand, Canada, Israel, Japan, Spain and Sweden), the incidence rate of HDP was $13 \%$ (ranging from $10 \cdot 3$ to $16 \cdot 4 \%)^{(3)}$.
Preterm birth (PTB) is the premature delivery of a neonate before 37 weeks of gestation ${ }^{(4)}$. PTB is most common in low- and middle-income countries and is one of the leading causes of direct neonatal deaths and complications $^{(4)}$, responsible for more than $50 \%$ of neonatal mortality in $2010^{(5)}$. According to a systematic analysis and estimation of PTB, the rate of PTB was $11 \%$ in 2010 globally, ranging from $5 \%$ in European countries to $18 \%$ in some African countries ${ }^{(6)}$. Likewise, low birth weight (LWB), which refers to a newborn birth weight of less than $2.5 \mathrm{~kg}$, is 
common (15\%). High rates are reported in many developing countries, especially South Asia (25\%), sub-Saharan Africa $(12 \%)^{(7)}$, Pakistan (35\%), Nepal (30\%) and Jordan $(22 \%)^{(8)}$.

Evidence has shown that dietary patterns have an influence on adverse pregnancy and birth outcomes ${ }^{(9,10)}$. When individuals consume foods, they consume a combination of nutrients, not single nutrients ${ }^{(11)}$. The whole diet with its expected synergistic effects may have a greater influence on the occurrence of health outcomes than single nutrients ${ }^{(11)}$. Hence, it appears more complete to examine the effect of the whole diet by applying a more all-inclusive method of dietary pattern analysis, because dietary patterns evaluate the usual diet as one complete dietary exposure $^{(12,13)}$.

Dietary pattern analysis aims to assess the usual foods consumed as one overall dietary exposure ${ }^{(12,14)}$. Dietary patterns are defined as the quantities, proportions, variety or combinations of different foods and beverages in diets and the frequency with which they are regularly consumed $^{(15)}$. Dietary patterns can be determined by three approaches. The first is the a priori approach, which constructs dietary scores or indices based on predefined dietary recommendations ${ }^{(12,14,16)}$. The second is the a posteriori approach, which identifies data-driven dietary patterns using statistical methods (cluster analysis and principal component analysis (PCA) $)^{(12,14,16)}$. The third approach consists of hybrid methods such as reduced rank regression, which combine aspects of the a priori and a posteriori approaches ${ }^{(16)}$.

Previous studies have indicated that dietary patterns during pregnancy have a varied effect on maternal health and pregnancy outcomes such as $\operatorname{HDP}^{(10,17)}, \mathrm{GDM}^{(18,19)}$, $\mathrm{PTB}^{(9,20,21)}$ and $\mathrm{LBW}^{(22)}$. For HDP, intake of vegetables, legumes, nuts, tofu, rice, pasta, rye bread, fish, milk, green leafy vegetables and pulses/beans was associated with a lower odds of pre-eclampsia/eclampsia ${ }^{(10,23)}$, while the consumption of meat and potatoes, processed meat, sweet drinks and salty snacks increased the likelihood of preeclampsia ${ }^{(10,24,25)}$. Other studies have reported contradictory findings; a cohort study in the USA ${ }^{(17)}$ reported that a higher Alternate Healthy Eating Index (AHEI) score comprising vegetables, fruit, fibre, trans fat, high PUFA:SFA, folate, Ca and Fe from foods was not associated with pre-eclampsia. For GDM, a Western dietary pattern (high intake of red meat, processed meat, refined grain products, sweets, French fries and pizza) among pregnant women in the USA ${ }^{(26)}$, a pasta-cheese-processed-meat pattern ${ }^{(18)}$ in a singaporean population and a sweet and seafood pattern in China ${ }^{(19)}$ have been associated with increased odds of GDM.

Regarding the birth outcomes, a 'prudent' dietary pattern with a high intake of vegetables, fruits, oils, water (beverage), wholegrain cereals and fibre-rich breads was associated with a reduced occurrence of $\mathrm{PTB}^{(9)}$. In contrast, a Western pattern (salty and sweet snacks, white bread, desserts and processed meat products) ${ }^{(9)}$ and a Mediterranean diet with a high intake of fish, fruit, vegetables, olive/canola oil, and a low intake of red meat and coffee had no effect on PTB ${ }^{(20)}$. Contrary to this, in a Danish birth cohort study, the odds of PTB increased in women who adhered to a Western pattern (high in meat and fats and low in fruits and vegetables) ${ }^{(21)}$. A study from the USA ${ }^{(27)}$ revealed that birth weight and fetal growth were not associated with the maternal AHEI score (high intakes of vegetables, fruit, whole grains, nuts and legumes, longchain (n-3) fats, polyunsaturated fats, folate, $\mathrm{Ca}$ and $\mathrm{Fe}$ ).

Current epidemiological studies show some evidence for an association between dietary patterns and adverse pregnancy and birth outcomes. However, the findings are inconsistent and there is a need to identify which dietary patterns could have health benefits for pregnant women in preventing adverse pregnancy and birth outcomes. Therefore, our aim was to determine the association between dietary patterns during pregnancy and the risk of pregnancy (HDP, GDM) and birth (PTB and LBW) outcomes through a systematic review and meta-analysis.

\section{Methods}

\section{Search strategy}

Seven databases were searched, including MEDLINE, EMBASE, CINAHL, Scopus, Cochrane Library, Web of Science, and Maternity and Infant Care. The reference lists of all previous articles were hand-searched.

The following terms, words and combinations of words were searched: ('diet' OR 'nutrition' OR 'food pattern' OR 'meal pattern' OR 'eating practice' OR 'food intake' OR 'food habits' OR 'eating behaviour' OR 'dietary pattern' OR 'dietary diversity score' AND 'pregnancy' OR 'pregnant women' OR 'gravid' OR 'gestation' OR 'prenatal care' OR 'antenatal care' AND 'gestational hypertension' OR 'pregnancy-induced hypertension' OR 'preeclampsia' OR 'pre-eclampsia' OR 'low birth weight' OR 'premature infant' OR 'premature birth' OR 'preterm birth' OR 'pregnancy in diabetics' OR 'gestational diabetes mellitus').

The search was comprised of free text words, title and Medical Subject Heading for outcomes, exposure and participants, as well as applying limits including English language and human subjects.

\section{Study selection}

The studies were screened by title and then by abstract by two reviewers (K.T.K., T.K.T.). The full texts of all selected studies were critically reviewed based on the inclusion/ exclusion criteria summarized in Table 1.

\section{Data extraction}

The following variables were extracted by two reviewers (K.T.K., T.K.T.): authors, publication year, study period, study design, settings/country, sample, dietary patterns with food details, dietary assessment methods and 
Table 1 Inclusion and exclusion criteria for the current systematic review and meta-analysis on maternal dietary patterns and risk of adverse pregnancy and birth outcomes

Inclusion criteria

- Pregnant women

- No date restrictions

- Original articles (randomized trials and observational studies)

- Dietary pattern as the exposure variable

- Included one or more of the following outcome variables: HDP, GDM, LBW, PTB

Exclusion criteria

- High-risk populations: women with heart diseases, diabetes, preeclampsia or gestational hypertension at baseline

- Unpublished papers

- Animal studies

- Brief communications, case series, editorials, review studies

- Studies that focused on single nutrients

HDP, hypertensive disorders of pregnancy (gestational hypertension, preeclampsia and eclampsia); GDM, gestational diabetes mellitus; LBW, low birth weight; PTB, preterm birth.

periods, main outcomes (HDP, GDM, LBW and PTB) and adjustment for confounding factors.

\section{Quality assessments}

The quality of selected full-text articles was rated by two reviewers independently (K.T.K., T.K.T.) using the Academy of Nutrition and Dietetics quality appraisal tool ${ }^{(28)}$. This tool has four relevance questions and ten validity questions. The validity questions appraise the selection, comparability of groups, assessment of exposures or outcomes and statistical analysis for each study separately ${ }^{(28)}$. The validity of a study is assessed as the responses to all relevant questions being 'yes'. The response for all validity questions is 'yes' if the criterion was fulfilled, 'no' if not fulfilled, 'unclear' if not precisely stated and ' $\mathrm{N} / \mathrm{A}$ ' (not applicable) if the criterion does not apply to the $\operatorname{articles}^{(28)}$. The rating scores of studies were positive (+) if the responses to the validity questions were 'yes' for six or more responses (including all four relevance questions). If the articles did not fulfil the relevance criterion of selection, comparability of groups and measurement of exposures or outcomes, the rating score was neutral $(\varnothing)$ and if the responses for the validity questions are 'no' or 'unclear' for six or more responses, a negative (-) rating score was given ${ }^{(28)}$.

\section{Statistical analysis}

The data were entered into a Microsoft ${ }^{\circledR}$ Excel spreadsheet version 16 and exported to the statistical software package Stata version 13 for analysis. The OR was used as a measure of effect estimate. If an incidence of outcome variable was less than or equal to $20 \%$, the risk ratio (RR) and OR were pooled together in the meta-analysis; otherwise RR was converted to OR using the proposed methods of Zhang and $\mathrm{Yu}^{(29)}$ and Cochrane ${ }^{(30)}$. If the studies did not report $\mathrm{OR} / \mathrm{RR}$ but reported the coefficient $(\beta)$ of the regression, it was converted into OR/RR by exponentiation of the coefficient (i.e. $\mathrm{OR}=\exp (\beta))^{(31)}$.

Some articles reported OR/RR based on different references. Some used lower adherence to dietary patterns, while some used good adherence. To make this consistent and unify all results using either the higher or lower group as reference, the new OR/RR was calculated by taking the reciprocal of the reported OR/RR. The lower limit of the new OR/RR is the reciprocal of the upper limit of the old $\mathrm{OR} / \mathrm{RR}$ and the upper limit of the new $\mathrm{OR} / \mathrm{RR}$ is reciprocal of the lower limit of the old OR/RR ${ }^{(32)}$.

The random-effects model was used for calculating pooled estimates. Statistical heterogeneity was evaluated by Cochran's $Q$ test $\left(I^{2}\right)$, which shows the amount of heterogeneity between studies. An $I^{2}$ value reflects betweenstudy variation (values of 25,50 and $75 \%$ refer to low, medium and high variation, respectively) ${ }^{(33)}$.

Subgroup analyses were conducted to detect potential sources of heterogeneity. The possible effects of betweenstudy variance of dietary assessment methods (dietary diversity score (DDS), Mediterranean diet score (MDS), PCA) and dietary assessment periods/trimesters (first trimester (1st-12th weeks), second trimester (13th-27th weeks), third trimester (28th-40th weeks)) were assessed.

Dietary patterns detected in each study were different regarding to the country of origin and the approaches used for identifying dietary patterns; however, they had similarities among commonly consumed food items. For instance, most articles identified a prudent, traditional, Mediterranean or healthy dietary pattern which commonly consisted of whole grains, nuts legumes/pulses, vegetables/fruits and fish. These studies were grouped together and analysed by labelling them as 'healthy dietary pattern'.

Similarly, those patterns comprised mostly of refined grains, processed meats or snacks, high-sugar and high-fat dairy products, eggs and white potatoes were grouped together, labelled as the 'Western dietary pattern' and then analysed.

Using the available articles, pooled estimates were determined for the effect of the healthy pattern on HDP, GDM, PTB and LBW. Likewise, meta-analysis was performed for a Western dietary pattern and GDM, HDP and PTB.

\section{Results}

\section{Identified studies}

Our search identified 6291 records after removal of duplicates. One hundred articles were identified for fulltext review, with twenty-one articles incorporated in the systematic review and meta-analysis (Fig. 1).

\section{Study characteristics}

Of the twenty-one articles included, the majority ( $n$ 15) were conducted in developed countries, with the 


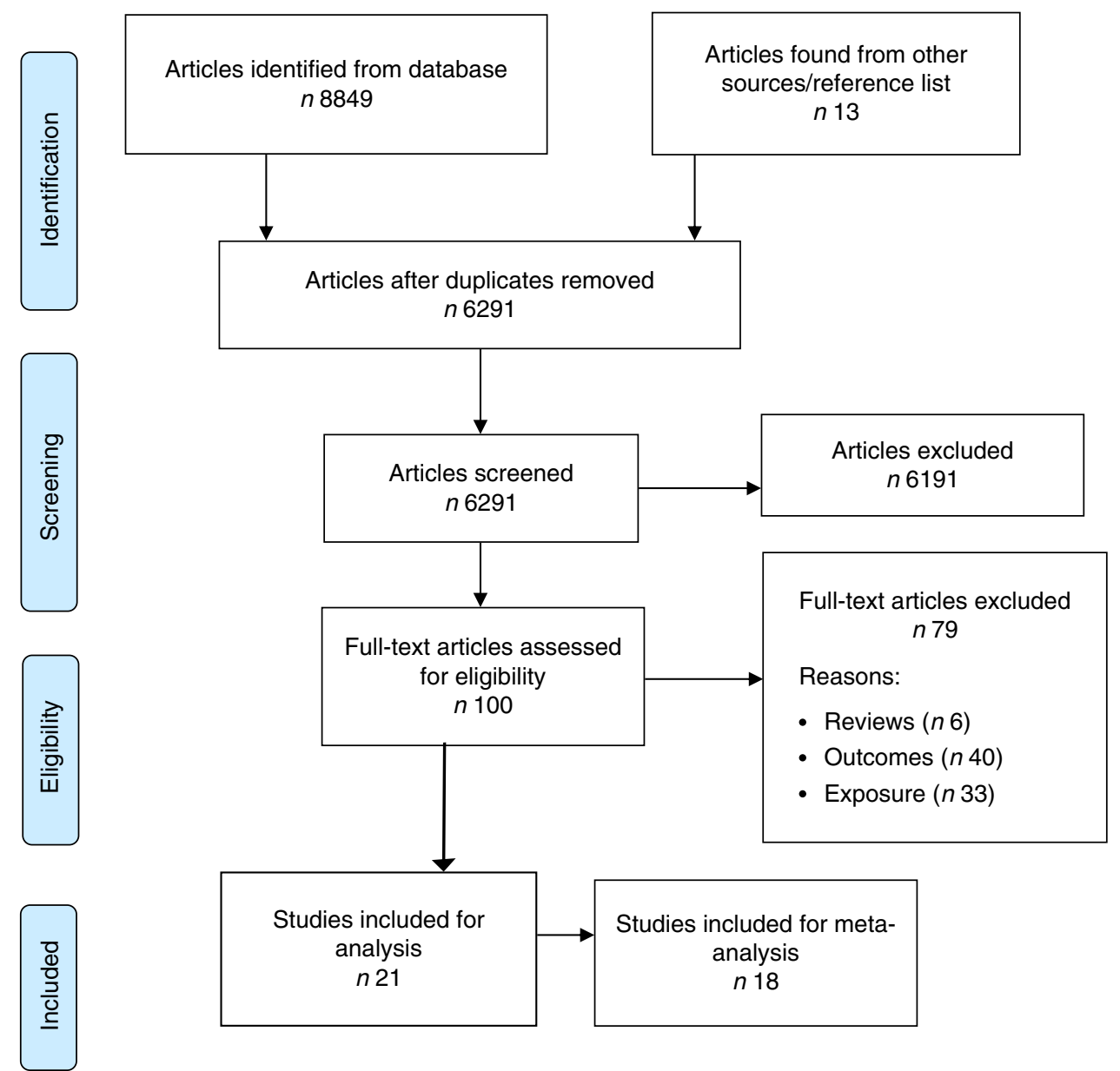

Fig. 1 (colour online) Flowchart of the study selection process for the current systematic review and meta-analysis on maternal dietary patterns and risk of adverse pregnancy and birth outcomes

remainder in developing countries. Out of all included articles, eighteen were cohort studies and three were cross-sectional studies. The articles were published between 2008 and 2016. The sample in each study ranged from $168^{(34)}$ to $66000^{(9)}$ with 302450 pregnant women in total. In the included articles, six reported the effect of dietary patterns on $\mathrm{HDP}^{(10,25,35-38)}$, six reported on $\mathrm{GDM}^{(18,19,34,39-41)}$, nine reported on $\operatorname{PTB}^{(9,20,21,36,42-46)}$ and two reported on $\mathrm{LBW}^{(46,47)}$ (Table 2).

Most of the articles ( $n$ 15) used an FFQ $^{(9,10,19-21,25,35,36,38,40,41,43-45,47)}$ as the method of dietary assessment, five studies used a $24 \mathrm{~h}$ recall $^{(18,37,39,42,46)}$, and one used a four-day food record $^{(34)}$ to assess the dietary intake. Various types of approaches were used to identify dietary patterns. Most studies applied the a posteriori approach (PCA; $n$ $13)^{(9,10,18,19,21,25,34,35,38,41-43,47)}$; seven studies used the $a$ priori method, $\operatorname{DDS}^{(37,46)}$ or $\operatorname{MDS}^{(20,40,44,45)}$ or New Nordic Diet $(\mathrm{NND})^{(36)}$; and one study used the rank reduced regression method ${ }^{(39)}$ to identify the dietary pattern of the women (Table 2). All studies had a positive score for the quality assessment.
The association of dietary patterns with adverse pregnancy outcomes (bypertensive disorders of pregnancy and gestational diabetes mellitus)

Dietary pattern and bypertensive disorders of pregnancy Six articles ${ }^{(10,25,35-38)}$ assessed the association between dietary pattern and HDP. These articles identified a range of different dietary patterns like healthy, traditional, Mediterranean and Western patterns, and therefore the results could not be pooled in meta-analysis except the healthy dietary pattern.

Healthy dietary pattern. Four studies ${ }^{(10,25,36,38)}$ were available for meta-analysis that reported the association between a healthy dietary pattern with a high intake of fruits, vegetables, whole-grain foods, fish and poultry and HDP. Based on this pooled analysis, study participants who adhered to a healthy dietary pattern were shown to have significantly lower odds of pre-eclampsia (OR $=0.78$, $95 \%$ CI $0.70,0 \cdot 86 ; I^{2}=39 \cdot 0 \%, P=0 \cdot 178$; Fig. 2 ).

However, one ${ }^{(37)}$ cross-sectional study in Tanzania indicated that having a high DDS (OR $=5 \cdot 84 ; 95 \%$ CI $2 \cdot 11$, $16 \cdot 15$ ) or a medium DDS (OR $=2 \cdot 54 ; 95 \%$ CI $1 \cdot 04,6 \cdot 16$ ) was associated with increased odds of gestational hypertension. On the contrary, in a cohort study, no association 
Table 2 Characteristics of the articles included in the current systematic review and meta-analysis on maternal dietary patterns and risk of adverse pregnancy and birth outcomes

\begin{tabular}{|c|c|c|c|c|c|c|c|c|c|}
\hline \multirow[b]{2}{*}{ Study } & \multirow[b]{2}{*}{$\begin{array}{l}\text { Study design; } \\
\text { period; country }\end{array}$} & \multirow[b]{2}{*}{$\begin{array}{c}\text { Sample } \\
(n)\end{array}$} & \multicolumn{2}{|c|}{ Dietary assessment } & \multirow{2}{*}{$\begin{array}{l}\text { Methods of } \\
\text { defining dietary } \\
\text { pattern }\end{array}$} & \multirow{2}{*}{$\begin{array}{l}\text { Dietary patterns } \\
\text { identified }\end{array}$} & \multirow[b]{2}{*}{ Main findings } & \multirow[b]{2}{*}{ Outcomes } & \multirow[b]{2}{*}{ Confounding factors } \\
\hline & & & Methods & $\begin{array}{l}\text { Trimester } \\
\text { (period) }\end{array}$ & & & & & \\
\hline $\begin{array}{l}\text { Brantsaeter } \\
\quad \text { et al. } \\
(2009)^{(10)}\end{array}$ & $\begin{array}{l}\text { Cohort; 2002- } \\
\text { 2006; Norway }\end{array}$ & 23423 & $\begin{array}{c}\text { 255-item } \\
\text { FFQ }^{*}\end{array}$ & $\begin{array}{l}\text { 2nd (17- } \\
\quad 22 \text { weeks) }\end{array}$ & PCA & $\begin{array}{l}\text { Vegetable; potato } \\
\text { and fish; cakes } \\
\text { and sweets; } \\
\text { processed food }\end{array}$ & $\begin{array}{l}\text { Vegetable (tertile } 3 v \text {. tertile } 1) \text { : } \\
\text { OR }=0.72(95 \% \mathrm{Cl} 0.62,0.85) \\
\text { Processed food (tertile } 3 \text { v. tertile } \\
\text { 1): OR }=1.21(95 \% \mathrm{Cl} 1.03, \\
\text { 1.42) } \\
\text { Potato and fish (tertile } 3 \text { v. tertile } \\
\text { 1): OR }=1.00(95 \% \mathrm{Cl} 0.84, \\
1.18)\end{array}$ & PE & $\begin{array}{l}\text { BMI, education, age, smoking, } \\
\text { height, education status, } \\
\text { hypertension prior to } \\
\text { pregnancy, TEI, dietary } \\
\text { supplement use }\end{array}$ \\
\hline $\begin{array}{l}\text { Eshriqui } \\
\text { et al. } \\
\qquad(2016)^{(35)}\end{array}$ & $\begin{array}{l}\text { Cohort; 2009- } \\
\text { 2012; Brazil }\end{array}$ & 299 & $\begin{array}{l}\text { Eighty-two } \\
\text { item FFQ* }\end{array}$ & $\begin{array}{l}\text { 3rd (28- } \\
\quad 38 \text { weeks) }\end{array}$ & PCA & $\begin{array}{l}\text { Healthy; processed; } \\
\text { common Brazilian }\end{array}$ & 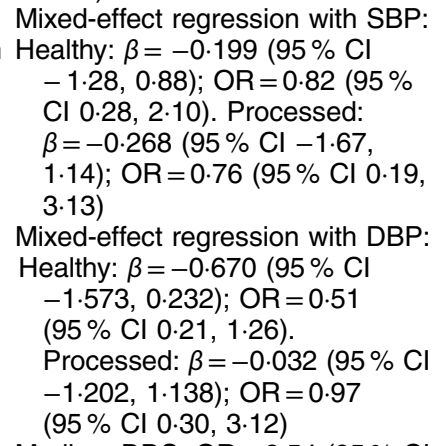 & $\begin{array}{c}\text { Blood } \\
\text { pressure } \\
\text { (SBP \& DBP) }\end{array}$ & $\begin{array}{l}\text { Age, BMI, education, parity, } \\
\text { TEl }\end{array}$ \\
\hline $\begin{array}{l}\text { Mwanri et al. } \\
(2015)^{(37)}\end{array}$ & $\begin{array}{l}\text { Cross-sectional; } \\
\text { 2011-2012; } \\
\text { Tanzania }\end{array}$ & 910 & $\begin{array}{l}\text { Sixteen-food- } \\
\text { group } 24 \mathrm{~h} \\
\text { recall }^{*}\end{array}$ & $\begin{array}{l}\text { 2nd \& 3rd (20- } \\
\quad 36 \text { weeks) }\end{array}$ & DDS & Sixteen food groups & $\begin{array}{l}\text { Medium DDS: OR=2.54 }(95 \% \mathrm{Cl} \\
1.04,6 \cdot 16) \\
\text { High DDS: OR }=5.84(95 \% \mathrm{Cl} \\
2 \cdot 11,16 \cdot 15)\end{array}$ & $\begin{array}{l}\text { Hypertension } \\
\text { during } \\
\text { pregnancy }\end{array}$ & $\begin{array}{l}\text { Residence, age, gestational } \\
\text { age, MUAC, parity, GDM, } \\
\text { education, PA }\end{array}$ \\
\hline $\begin{array}{l}\text { Timmermans } \\
\text { et al. } \\
(2011)^{(25)}\end{array}$ & $\begin{array}{l}\text { Prospective } \\
\text { cohort; the } \\
\text { Netherlands }\end{array}$ & 3187 & $\begin{array}{c}\text { 293-item } \\
\text { FFQ }^{*}\end{array}$ & $\begin{array}{l}\text { All (median } \\
13.5 \text { weeks) }\end{array}$ & PCA & $\begin{array}{l}\text { Mediterranean diet } \\
\text { pattern (MDP); } \\
\text { traditional dietary } \\
\text { pattern }\end{array}$ & $\begin{array}{l}\text { For PE: } \\
\text { Low adherence to MDP: OR }=1.2 \\
\text { (95\% } \mathrm{Cl} 0 \cdot 6,2 \cdot 3) \text {; adherence to } \\
\text { MDP: OR }=0.83 \text { (95\% } \mathrm{Cl} 0.43 \text {, } \\
\text { 1.60); adherence to traditional: } \\
\text { OR }=1 \cdot 1(0.6,2 \cdot 1) \\
\text { For GHTN: } \\
\text { Low adherence to MDP: OR }=1.3 \\
\text { (95\% } \mathrm{Cl} 0.9,1.9) \text {; adherence to } \\
\text { MDP: OR }=0.77(95 \% \mathrm{Cl} 0.53 \text {, } \\
\text { 1.11); adherence to traditional: } \\
\text { OR }=1.3(95 \% \mathrm{Cl} 0.9,1.9)\end{array}$ & PE \& GHTN & $\begin{array}{l}\text { Maternal BMI, maternal age, } \\
\text { parity, educational level, } \\
\text { smoking, vomiting, } \\
\text { preconception folic acid use }\end{array}$ \\
\hline $\begin{array}{l}\text { Torjusen } \\
\text { et al. } \\
(2014)^{(38)}\end{array}$ & $\begin{array}{l}\text { Cohort; 2002- } \\
\text { 2008; Norway }\end{array}$ & 28192 & $\begin{array}{l}\text { Six-food- } \\
\quad \text { group FFQ }\end{array}$ & $\begin{array}{l}\text { 2nd (17- } \\
22 \text { weeks) }\end{array}$ & PCA & $\begin{array}{l}\text { Healthy pattern; } \\
\text { organic } \\
\text { vegetables } \\
\text { pattern }\end{array}$ & $\begin{array}{l}\text { Healthy pattern, tertile } 3 \mathrm{v} \text {. tertile } \\
\text { 1: OR }=0.74(95 \% \mathrm{Cl} 0.64 \text {, } \\
0.85) \\
\text { Organic vegetables, tertile } 3 \mathrm{v} \text {. } \\
\text { tertile } 1: \text { OR }=0.79(95 \% \mathrm{Cl} \\
0.62,0.99)\end{array}$ & PE & $\begin{array}{l}\text { Hypertension prior to } \\
\text { pregnancy, pre-pregnant } \\
\text { BMl, height, age, education, } \\
\text { household income, smoking } \\
\text { in pregnancy, TEI, } \\
\text { gestational weight gain }\end{array}$ \\
\hline
\end{tabular}


Table 2 Continued

\begin{tabular}{|c|c|c|c|c|c|c|c|c|c|}
\hline \multirow[b]{2}{*}{ Study } & \multirow[b]{2}{*}{$\begin{array}{l}\text { Study design; } \\
\text { period; country }\end{array}$} & \multirow[b]{2}{*}{$\begin{array}{l}\text { Sample } \\
(n)\end{array}$} & \multicolumn{2}{|c|}{ Dietary assessment } & \multirow{2}{*}{$\begin{array}{c}\text { Methods of } \\
\text { defining dietary } \\
\text { pattern }\end{array}$} & \multirow{2}{*}{$\begin{array}{l}\text { Dietary patterns } \\
\text { identified }\end{array}$} & \multirow[b]{2}{*}{ Main findings } & \multirow[b]{2}{*}{ Outcomes } & \multirow[b]{2}{*}{ Confounding factors } \\
\hline & & & Methods & $\begin{array}{l}\text { Trimester } \\
\text { (period) }\end{array}$ & & & & & \\
\hline $\begin{array}{l}\text { Hillesund } \\
\text { et al. } \\
\quad(2014)^{(36)}\end{array}$ & Cohort; Norway & 72072 & $\begin{array}{c}\text { 255-item } \\
\mathrm{FFQ}^{*}\end{array}$ & 25 weeks & NND score & $\begin{array}{l}\text { New Nordic dietary } \\
\text { index (NND) }\end{array}$ & $\begin{array}{l}\text { With high NND score: } \\
\text { Risk of PE: OR=0.86 (95\% Cl } \\
0.78,0.95) ; \text { risk of early PE: } \\
\text { OR }=0.71(95 \% \mathrm{Cl} 0.52,0.96) \text {; } \\
\text { risk of PTB: OR }=0.91(95 \% \mathrm{Cl} \\
0.80,1.30)\end{array}$ & PE \& PTB & $\begin{array}{l}\text { Maternal age, height, pre- } \\
\text { pregnancy BMI, parity, } \\
\text { education, smoking status, } \\
\text { exercise during pregnancy, } \\
\text { chronic hypertension, } \\
\text { diabetes, marital status, } \\
\text { energy intake }\end{array}$ \\
\hline $\begin{array}{l}\text { Dayeon et al. } \\
(2015)^{(39)}\end{array}$ & $\begin{array}{l}\text { Cross-sectional; } \\
\text { USA }\end{array}$ & 253 & $\begin{array}{l}\text { Eight-food- } \\
\quad \text { group } 24 \mathrm{~h} \\
\text { recall }\end{array}$ & $\begin{array}{l}\text { All (avg. } \\
20 \text { weeks) }\end{array}$ & RRR & $\begin{array}{l}\text { 'High refined grains', } \\
\text { 'high nuts, seeds, } \\
\text { fat and } \\
\text { soyabeans, low } \\
\text { milk', 'high added } \\
\text { sugar and organ } \\
\text { meats', 'low fruits, } \\
\text { vegetables and } \\
\text { seafood' }\end{array}$ & $\begin{array}{l}\text { 'High refined grains' pattern: } \\
\text { OR }=4.9(95 \% \mathrm{Cl} 1.4,17 \cdot 0) \\
\text { 'High nuts, seeds, fat and } \\
\text { soyabeans, low milk' pattern: } \\
\text { OR }=7.5(95 \% \mathrm{Cl} 1.8,32 \cdot 3) \\
\text { 'High added sugar and organ } \\
\text { meats' pattern: OR }=22.3(95 \% \\
\text { Cl } 3.9,127.4)\end{array}$ & GDM & $\begin{array}{l}\text { Age, race/ethnicity, family } \\
\text { poverty income ratio, } \\
\text { education, marital status, } \\
\text { energy intake, pre- } \\
\text { pregnancy BMI, gestational } \\
\text { weight gain, log-transformed } \\
\text { CRP }\end{array}$ \\
\hline $\begin{array}{l}\text { De Seymour } \\
\text { et al. } \\
(2016)^{(18)}\end{array}$ & $\begin{array}{l}\text { Multi-ethnic } \\
\text { Asian cohort; } \\
\text { Singapore }\end{array}$ & 909 & $\begin{array}{l}\text { Sixty-eight- } \\
\text { food-group } \\
24 \mathrm{~h} \text { recall* }\end{array}$ & $\begin{array}{l}\text { 2nd \& 3rd (26- } \\
\quad 28 \text { weeks) }\end{array}$ & PCA & $\begin{array}{l}\text { Three patterns: } \\
\text { vegetable-fruit- } \\
\text { rice-based-diet; } \\
\text { seafood-noodle- } \\
\text { based-diet; } \\
\text { pasta-cheese-- } \\
\text { processed-meat } \\
\text { diet }\end{array}$ & $\begin{array}{l}\text { Vegetable-fruit-rice-based-diet: } \\
\text { OR }=1 \cdot 10 \text { (95\% Cl 0.90, } 1.35) \\
\text { Seafood-noodle-based-diet: } \\
\text { OR }=0.74(95 \% \mathrm{Cl} 0.59,0.93) \\
\text { Pasta-cheese-processed-meat } \\
\text { diet: OR }=0.96(95 \% \mathrm{Cl} 0.79 \\
1.17)\end{array}$ & GDM & $\begin{array}{l}\text { Energy intake, pregnancy BMI, } \\
\text { birth order, smoking, alcohol } \\
\text { intake, age, ethnicity, } \\
\text { education, previous GDM, } \\
\text { family history of diabetes, } \\
\text { household monthly income, } \\
\text { other dietary patterns }\end{array}$ \\
\hline $\begin{array}{l}\text { He et al. } \\
\quad(2015)^{(19)}\end{array}$ & $\begin{array}{l}\text { Prospective } \\
\text { cohort, China }\end{array}$ & 3063 & $\begin{array}{l}\text { Sixty-four- } \\
\text { item FFQ* }\end{array}$ & $\begin{array}{l}\text { 2nd (24- } \\
27 \text { weeks) }\end{array}$ & PCA & $\begin{array}{l}\text { Four dietary } \\
\text { patterns: } \\
\text { vegetable pattern; } \\
\text { protein-rich food } \\
\text { pattern; prudent } \\
\text { pattern; sweets } \\
\text { and seafood } \\
\text { pattern }\end{array}$ & $\begin{array}{l}\text { Vegetable pattern: RR }=0.79 \\
(95 \% \mathrm{Cl} 0.64,0.97) \\
\text { Sweets and seafood pattern: } \\
\text { RR }=1.23(95 \% \mathrm{Cl} 1.02,1.49) \\
\text { Protein-rich food pattern: } \\
\text { RR }=0.95(95 \% \mathrm{Cl} 0.78,1.16) \\
\text { Prudent pattern: } \mathrm{RR}=1.00(95 \% \\
\text { Cl } 0.82,1.22)\end{array}$ & GDM & $\begin{array}{l}\text { Maternal age, education level, } \\
\text { monthly income, parity, pre- } \\
\text { pregnancy BMI, family } \\
\text { history of diabetes }\end{array}$ \\
\hline $\begin{array}{l}\text { Karamanos } \\
\text { et al. } \\
(2014)^{(40)}\end{array}$ & $\begin{array}{l}\text { Prospective } \\
\text { cohort; Jan } \\
\text { 2010-Jul } \\
\text { 2011; ten } \\
\text { Mediterranean } \\
\text { countries }\end{array}$ & 1076 & $\begin{array}{l}\text { Seventy- } \\
\text { eight-item } \\
\text { FFQ }^{\star}\end{array}$ & $\begin{array}{l}\text { 2nd \& 3rd (24- } \\
\quad 32 \text { weeks) }\end{array}$ & MDS & $\begin{array}{l}\text { Mediterranean diet } \\
\text { index }\end{array}$ & $\begin{array}{l}\text { Mediterranean diet: } \mathrm{OR}=0.618 \\
\quad(95 \% \mathrm{Cl} 0.401,0.950)\end{array}$ & GDM & $\begin{array}{l}\text { Age, BMI, diabetes in the } \\
\text { family, weight gain, energy } \\
\text { intake }\end{array}$ \\
\hline $\begin{array}{l}\text { Nascimento } \\
\text { et al. } \\
(2016)^{(41)}\end{array}$ & $\begin{array}{l}\text { Prospective } \\
\text { cohort; Nov } \\
\text { 2011-Feb } \\
\text { 2014; Spain }\end{array}$ & 841 & $\begin{array}{l}\text { Eighty-one- } \\
\text { item FFQ* }\end{array}$ & $\begin{array}{l}\text { 2nd (15- } \\
20 \text { weeks) }\end{array}$ & PCA & $\begin{array}{l}\text { Three patterns: } \\
\text { traditional pattern; } \\
\text { vegetable and } \\
\text { Western pattern; } \\
\text { mixed pattern }\end{array}$ & $\begin{array}{l}\text { High tertile } v \text {. low tertile }(3 \mathrm{v} .1) \text { : } \\
\text { Traditional pattern: RR }=0.88 \\
(95 \% \mathrm{Cl} 0.49,1.58) \\
\text { Mixed pattern: RR }=0.93(95 \% \mathrm{Cl} \\
0.51,1.71) \\
\text { Vegetable and Western pattern: } \\
\mathrm{RR}=0.78(95 \% \mathrm{Cl} 0.43,1.43)\end{array}$ & GDM & $\begin{array}{l}\text { BMI, age, education, monthly } \\
\text { income, family history of } \\
\text { diabetes, parity }\end{array}$ \\
\hline
\end{tabular}




\begin{tabular}{|c|c|c|c|c|c|c|c|c|c|}
\hline \multirow[b]{2}{*}{ Study } & \multirow[b]{2}{*}{$\begin{array}{l}\text { Study design; } \\
\text { period; country }\end{array}$} & \multirow[b]{2}{*}{$\begin{array}{l}\text { Sample } \\
(n)\end{array}$} & \multicolumn{2}{|c|}{ Dietary assessment } & \multirow{2}{*}{$\begin{array}{l}\text { Methods of } \\
\text { defining dietary } \\
\text { pattern }\end{array}$} & \multirow{2}{*}{$\begin{array}{l}\text { Dietary patterns } \\
\text { identified }\end{array}$} & \multirow[b]{2}{*}{ Main findings } & \multirow[b]{2}{*}{ Outcomes } & \multirow[b]{2}{*}{ Confounding factors } \\
\hline & & & Methods & $\begin{array}{l}\text { Trimester } \\
\text { (period) }\end{array}$ & & & & & \\
\hline $\begin{array}{l}\text { Tryggvadottir } \\
\text { et al. } \\
(2016)^{(34)}\end{array}$ & $\begin{array}{l}\text { Prospective } \\
\text { cohort; Apr } \\
\text { 2012-Oct } \\
\text { 2013; Iceland }\end{array}$ & 168 & $\begin{array}{l}\text { Eighteen- } \\
\text { food-group } \\
\& 4 \mathrm{~d} \\
\text { weighed } \\
\text { food record }\end{array}$ & $\begin{array}{l}\text { 2nd (19- } \\
24 \text { weeks) }\end{array}$ & PCA & Prudent pattern & $\begin{array}{l}\text { Adhering to the prudent pattern: } \\
\text { OR }=0.44(95 \% 0.21,0.90)\end{array}$ & GDM & $\begin{array}{l}\text { Age, parity, pre-pregnancy } \\
\text { weight, energy intake, } \\
\text { weekly weight gain, total } \\
\text { metabolic equivalents of } \\
\text { task }\end{array}$ \\
\hline $\begin{array}{l}\text { Chia et al. } \\
\qquad(2016)^{(42)}\end{array}$ & $\begin{array}{l}\text { Cohort study; } \\
\text { 2009-2010; } \\
\text { Singapore }\end{array}$ & 923 & $\begin{array}{l}\text { Sixty-eight- } \\
\text { food-group } \\
24 \mathrm{~h} \text { recalls } \\
\text { and } 3 \mathrm{~d} \\
\text { food } \\
\text { diaries }\end{array}$ & $\begin{array}{l}\text { 2nd \& 3rd (26- } \\
28 \text { weeks) }\end{array}$ & PCA & $\begin{array}{l}\text { Vegetable, fruit and } \\
\text { white rice; } \\
\text { seafood and } \\
\text { noodle; pasta, } \\
\text { cheese and } \\
\text { processed meat }\end{array}$ & $\begin{array}{l}\text { Adherence to vegetable, fruit and } \\
\text { white rice pattern: OR=0.67 } \\
\text { ( } 95 \% \mathrm{Cl} 0.50,0.91) \\
\text { Adherence to seafood and noodle } \\
\text { pattern: OR }=1.27 \text { ( } 95 \% \mathrm{Cl} \\
0.93,1.74) \\
\text { Adherence to pasta, cheese and } \\
\text { processed meat pattern: } \\
\text { OR }=0.79(95 \% \mathrm{Cl} 0.55,1.12)\end{array}$ & 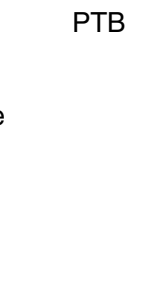 & $\begin{array}{l}\text { Infant sex, birth order, maternal } \\
\text { TEl, maternal age, ethnicity, } \\
\text { pre-pregnancy BMI, weight } \\
\text { gain until } 26-28 \text { week of } \\
\text { gestation, height, GDM } \\
\text { status, educational status, } \\
\text { alcohol use, smoking during } \\
\text { pregnancy, other dietary } \\
\text { patterns }\end{array}$ \\
\hline $\begin{array}{l}\text { Englund- } \\
\text { Ogge et al. } \\
(2014)^{(9)}\end{array}$ & $\begin{array}{l}\text { Prospective } \\
\text { cohort; 2002- } \\
\text { 2008; Norway }\end{array}$ & 66000 & $\begin{array}{c}\text { 255-item } \\
\text { FFQ* }^{*}\end{array}$ & $\begin{array}{l}\text { 2nd (17- } \\
22 \text { weeks) }\end{array}$ & PCA & $\begin{array}{l}\text { 'Prudent'; 'Western'; } \\
\text { 'traditional' }\end{array}$ & $\begin{array}{l}\text { Prudent: } R R=0.88(95 \% \mathrm{Cl} 0.80 \text {, } \\
0.97) \\
\text { Western: } R R=1.02(95 \% \mathrm{Cl} 0.92 \text {, } \\
1.13) \\
\text { Traditional: } R R=0.91(95 \% \mathrm{Cl} \\
0.83,0.99)\end{array}$ & PTB & $\begin{array}{l}\text { Maternal age, pre-pregnancy } \\
\text { BMI, height, parity, TEI, } \\
\text { maternal education, marital } \\
\text { status, smoking, previous } \\
\text { preterm delivery, household } \\
\text { income, other dietary } \\
\text { patterns }\end{array}$ \\
\hline $\begin{array}{l}\text { Haugen et al. } \\
(2008)^{(20)}\end{array}$ & Cohort; Norway & 569 & $\begin{array}{l}\text { 255-item } \\
\mathrm{FFQ}^{*}\end{array}$ & $\begin{array}{l}\text { 2nd (18- } \\
22 \text { weeks) }\end{array}$ & MDS & $\begin{array}{l}\text { Mediterranean diet } \\
\text { criteria }\end{array}$ & $\begin{array}{l}\text { Mediterranean diet criteria } 5 \text { v. } 0: \\
\quad \mathrm{OR}=0.73(95 \% \mathrm{Cl} 0.32,1.68)\end{array}$ & PTB & $\begin{array}{l}\text { Parity, BMI, maternal height, } \\
\text { SES; cohabitant status }\end{array}$ \\
\hline $\begin{array}{l}\text { Martin et al. } \\
\quad(2015)^{(43)}\end{array}$ & $\begin{array}{l}\text { Prospective } \\
\text { cohort; USA }\end{array}$ & 3143 & $\begin{array}{l}\text { Ninety-five- } \\
\text { item FFQ }\end{array}$ & $\begin{array}{l}\text { 2nd \& } 3 \text { rd }(26- \\
29 \text { weeks })\end{array}$ & $\begin{array}{l}\text { PCA and } \\
\text { DASH }\end{array}$ & $\begin{array}{l}\text { Factor 1; Factor 2; } \\
\text { Factor 3; Factor } 4\end{array}$ & $\begin{array}{l}\text { Factor 1: OR }=0.87(95 \% \mathrm{Cl} 0.60, \\
1.27) \\
\text { Factor } 2: \mathrm{OR}=1.53(95 \% \mathrm{Cl} 1.02, \\
2.30) \\
\text { Factor } 3: \text { OR }=1.55(95 \% \mathrm{Cl} 1.07, \\
2.24) \\
\text { Adherence to DASH diet: } \\
\text { OR }=0.59(95 \% \mathrm{Cl} 0.40,0.85)\end{array}$ & , & $\begin{array}{l}\text { Maternal age, race, maternal } \\
\text { pre-pregnancy BMl status, } \\
\text { educational level, household } \\
\text { income, parity, marital } \\
\text { status, smoking status, } \\
\text { energy intake }\end{array}$ \\
\hline $\begin{array}{l}\text { Rasmussen } \\
\text { et al. } \\
(2014)^{(21)}\end{array}$ & $\begin{array}{l}\text { Longitudinal } \\
\text { cohort; } \\
\text { Denmark }\end{array}$ & 59949 & $\begin{array}{c}\text { 360-item } \\
\mathrm{FFQ}^{*}\end{array}$ & $\begin{array}{l}\text { 2nd \& 3rd (avg. } \\
\quad 25 \text { weeks) }\end{array}$ & PCA & $\begin{array}{l}\text { Vegetable/prudent; } \\
\text { Western; Seafood }\end{array}$ & $\begin{array}{l}\text { Western pattern: OR }=1.30(95 \% \\
\text { Cl } 1.13,1.49) \\
\text { Vegetable/prudent pattern: } \\
\text { OR }=1.40(95 \% \mathrm{Cl} 0.80,1.62) \\
\text { Seafood pattern: OR }=0.90(95 \% \\
\mathrm{Cl} 0.72,1.11)\end{array}$ & PTB & $\begin{array}{l}\text { Maternal age, maternal height, } \\
\text { pre-pregnancy BMI, parity, } \\
\text { civil status, SES, smoking } \\
\text { during pregnancy }\end{array}$ \\
\hline $\begin{array}{l}\text { Zerfu et al. } \\
\quad(2016)^{(46)}\end{array}$ & $\begin{array}{l}\text { Prospective } \\
\text { cohort; } \\
\text { Ethiopia }\end{array}$ & 432 & $\begin{array}{l}\text { Nine-food- } \\
\text { group } 24 \mathrm{~h} \\
\text { WDDS }\end{array}$ & $\begin{array}{l}\text { 2nd \& 3rd (24- } \\
\quad 28 \text { weeks) }\end{array}$ & DDS & Nine food groups & $\begin{array}{l}\text { Low DDS: RR }=4.61(95 \% \mathrm{Cl} \\
\text { 2.31, 9.19) } \\
\text { High DDS: RR }=0.21(95 \% \mathrm{Cl} \\
0.11,0.43)\end{array}$ & РTB & $\begin{array}{l}\text { Age, height, MUAC, education, } \\
\mathrm{Hb} \text { level }\end{array}$ \\
\hline
\end{tabular}


Table 2 Continued

\begin{tabular}{|c|c|c|c|c|c|c|c|c|c|}
\hline \multirow[b]{2}{*}{ Study } & \multirow[b]{2}{*}{$\begin{array}{l}\text { Study design; } \\
\text { period; country }\end{array}$} & \multirow[b]{2}{*}{$\underset{(n)}{\text { Sample }}$} & \multicolumn{2}{|c|}{ Dietary assessment } & \multirow{2}{*}{$\begin{array}{l}\text { Methods of } \\
\text { defining dietary } \\
\text { pattern }\end{array}$} & \multirow{2}{*}{$\begin{array}{l}\text { Dietary patterns } \\
\text { identified }\end{array}$} & \multirow[b]{2}{*}{ Main findings } & \multirow[b]{2}{*}{ Outcomes } & \multirow[b]{2}{*}{ Confounding factors } \\
\hline & & & Methods & $\begin{array}{l}\text { Trimester } \\
\text { (period) }\end{array}$ & & & & & \\
\hline $\begin{array}{l}\text { Mikkelsen } \\
\text { et al. } \\
(2008)^{(44)}\end{array}$ & $\begin{array}{l}\text { Cohort; } \\
\text { Denmark }\end{array}$ & 35530 & $\begin{array}{l}\text { 360-item } \\
\text { FFQ }\end{array}$ & $\begin{array}{l}\text { 2nd \& 3rd (avg. } \\
\quad 25 \text { weeks) }\end{array}$ & MDS & $\begin{array}{l}\text { Mediterranean diet } \\
\text { criteria: } \\
\text { consumption of } \\
\text { fish twice/week; } \\
\text { intake of olive or } \\
\text { rapeseed oil; high } \\
\text { consumption of } \\
\text { fruits \& } \\
\text { vegetables (5/d or } \\
\text { more); meat } \\
\text { (other than poultry } \\
\text { and fish) at most } \\
\text { twice/week }\end{array}$ & $\begin{array}{l}\text { Mediterranean diet criteria } 5 \text { v. } 0 \text { : } \\
\text { OR }=0.61(95 \% \mathrm{Cl} 0.35,1.05) \\
\text { Mediterranean diet criteria } 5 \mathrm{v} .1- \\
\text { 4: } \mathrm{OR}=0.92(95 \% \mathrm{Cl} 0.69, \\
\text { 1.24) } \\
\text { Note: } 5 \mathrm{v} \text {. } 0 \text { means fulfilled } \geq 5 \mathrm{v} \text {. } \\
\text { no fulfilled criteria } \\
\end{array}$ & PTB & $\begin{array}{l}\text { Parity, BMI, maternal height, } \\
\text { SES, cohabitant status }\end{array}$ \\
\hline $\begin{array}{l}\text { Saunders } \\
\text { et al. } \\
\quad(2014)^{(45)}\end{array}$ & $\begin{array}{l}\text { Cohort; 2004- } \\
\text { 2007; French } \\
\text { Caribbean } \\
\text { island }\end{array}$ & $\begin{array}{l}\quad 728 \\
\text { ( } 710 \text { with } \\
\text { complete } \\
\text { data) }\end{array}$ & $\begin{array}{l}\text { 214-item } \\
\text { FFQ }\end{array}$ & $\begin{array}{l}\text { Days following } \\
\text { delivery }\end{array}$ & MDS & $\begin{array}{l}\text { Nine categories of } \\
\text { the Mediterranean } \\
\text { diet scale } \\
\text { (vegetables, } \\
\text { legumes, fruits } \\
\text { and nuts, cereals, } \\
\text { fish, meat and } \\
\text { poultry, dairy } \\
\text { products, alcohol, } \\
\text { fat) }\end{array}$ & $\begin{array}{l}\text { Adherence to Mediterranean diet: } \\
\text { OR }=0.9(95 \% \mathrm{Cl} 0.8,1 \cdot 0)\end{array}$ & PTB & $\begin{array}{l}\text { Maternal place of birth, marital } \\
\text { status, pre-pregnancy BMI, } \\
\text { maternal education, } \\
\text { enrolment site, weight gain } \\
\text { during pregnancy, energy } \\
\text { intake, maternal smoking } \\
\text { during pregnancy }\end{array}$ \\
\hline $\begin{array}{l}\text { Abubakari } \\
\text { and Jahn } \\
(2016)^{(47)}\end{array}$ & $\begin{array}{l}\text { Cross-sectional; } \\
\text { Ghana }\end{array}$ & 578 & $\begin{array}{l}\text { Fifty-five-item } \\
\text { FFQ* }^{*}\end{array}$ & $\begin{array}{l}\text { 2nd trimester } \\
\text { and 0- } \\
1 \text { month } \\
\text { post-birth }\end{array}$ & PCA & $\begin{array}{l}\text { Non-health } \\
\text { conscious; health } \\
\text { conscious }\end{array}$ & $\begin{array}{l}\text { Health conscious diet: OR }=0.23 \\
(95 \% \mathrm{Cl} 0.12,0.45) \\
\text { Non-health conscious diet: } \\
\text { OR }=1.04(95 \% \mathrm{Cl} 0.65,1.67) \\
\text { High DDS: OR }=0.10(95 \% \mathrm{Cl} \\
0.04,0.13)\end{array}$ & LBW & Gestational age \\
\hline $\begin{array}{l}\text { Zerfu } \\
\quad \text { et al. }\end{array}$ & Cohort; Ethiopia & 432 & $\begin{array}{l}\text { Nine-food- } \\
\text { group } 24 \mathrm{~h} \\
\text { WDDS }\end{array}$ & $\begin{array}{l}\text { 2nd \& 3rd (24- } \\
28 \text { weeks) }\end{array}$ & DDS & Nine food groups & $\begin{array}{l}\text { High DDS: RR }=2.06(95 \% \mathrm{Cl} \\
1.03,4.11)\end{array}$ & LBW & $\begin{array}{l}\text { Education, age, height, MUAC, } \\
\text { and } \mathrm{Hb} \text { level }\end{array}$ \\
\hline
\end{tabular}

WDDS, Women Dietary Diversity Score; avg., average; PCA, principal component analysis; DDS, dietary diversity score; RRR, reduced rank regression; MDS, Mediterranean diet score; DASH, Dietary Approaches to Stop

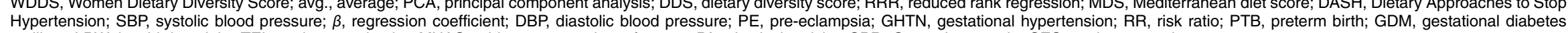
mellitus; LBW, low birth weight; TEI, total energy intake; MUAC, mid-upper arm circumference; PA, physical activity; CPR, C-reactive protein; SES, socio-economic status.

*Validated FFQ or recall was used. 


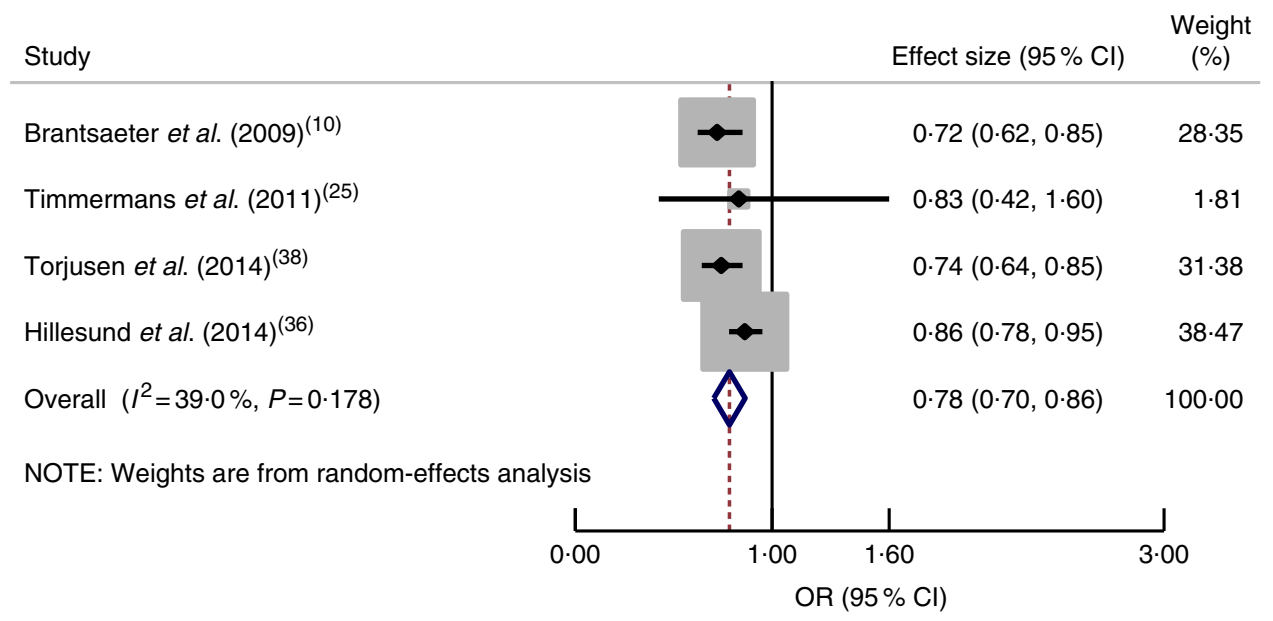

Fig. 2 (colour online) Forest plot for the pooled OR of the association between a healthy dietary pattern and pre-eclampsia. The study-specific OR and $95 \% \mathrm{Cl}$ are represented by the black diamond and the horizontal line, respectively; the area of the grey square is proportional to the specific-study weight to the overall meta-analysis. The centre of the blue open diamond and the red dashed vertical line represent the pooled OR; and the width of the blue open diamond represents the pooled $95 \% \mathrm{Cl}$

was observed between gestational hypertension and adherence to a Mediterranean pattern $(\mathrm{OR}=0 \cdot 77 ; 95 \% \mathrm{CI}$ $0.53,1 \cdot 11)$ or a traditional pattern $(\mathrm{OR}=1.3 ; 95 \% \mathrm{CI} 0.9$, $1.9)^{(25)}$. Likewise, a cohort study from Brazil ${ }^{(35)}$ revealed that adherence to a healthy dietary pattern did not have an effect on systolic blood pressure (OR $=0 \cdot 82 ; 95 \%$ CI $0 \cdot 28$, $2 \cdot 21$ ) or diastolic blood pressure (OR $=0 \cdot 94 ; 95 \%$ CI $0 \cdot 18$, $1 \cdot 28)$.

Western dietary pattern. In a cohort study in Norway ${ }^{(10)}$, a potato and fish dietary pattern (lean fish, cooked potatoes, processed fish; fish burgers, margarine, fish soufflé, meat spread, lean fish and poultry) was not associated with preeclampsia (OR = 1.00: $95 \%$ CI 0.84, 1·18). Likewise, a cohort study in Brazil ${ }^{(35)}$ reported that adherence to a processed food pattern was not significantly associated with the change in systolic blood pressure (OR $=0.76 ; 95 \%$ CI 0.19, $3 \cdot 13)$ and diastolic blood pressure $(\mathrm{OR}=0.97 ; 95 \% \mathrm{CI} 0.30$, 3.10) during pregnancy.

\section{Dietary pattern and gestational diabetes mellitus}

Healthy dietary pattern. Six studies ${ }^{(18,19,34,39-41)}$ assessed the effect of dietary patterns on GDM. A cohort study in Singapore ${ }^{(18)}$ indicated that a seafood-noodle-based diet was related with lower odds of GDM $(\mathrm{OR}=0.74$; $95 \%$ CI $0.59,0.93)$. However, higher $v$. lower adherence to a vegetable-fruit-rice-based diet $(\mathrm{OR}=1 \cdot 10 ; 95 \% \mathrm{CI}$ $0.90,1.35)$ and a pasta-cheese-processed-meat diet $(\mathrm{OR}=0.96 ; 95 \%$ CI $0.79,1.17)$ was not associated with GDM. Similarly, adherence to a traditional pattern $(\mathrm{RR}=0.88 ; 95 \% \mathrm{CI} 0.49,1.58)$ as well as adherence to a mixed pattern $(\mathrm{RR}=0.93 ; 95 \% \mathrm{CI} 0.51,1.71)$ was not associated with the incidence of GDM among Brazilian women $^{(41)}$.

The pooled estimate of a healthy dietary pattern on GDM was determined by using five studies $(18,19,34,40,41)$.
Based on this estimate, women who had higher adherence to a healthy dietary pattern had lower odds of GDM $(\mathrm{OR}=0.78 ; 95 \%$ CI 0.56 , 0.99), with significant heterogeneity detected between studies $\left(I^{2}=68 \cdot 6 \%, P=0 \cdot 013\right.$; Fig. 3(a)).

Western dietary pattern. Four studies ${ }^{(18,19,39,41)}$ were combined, showing no relationship between adherence to a Western dietary pattern and odds of GDM (OR =0.94; $95 \%$ CI $0.81,1.07)$ and no heterogeneity between studies $\left(I^{2}=0.0 \%, P=0.825\right.$; Fig. 3(b)).

A cross-sectional survey in the USA ${ }^{(39)}$ and a prospective cohort study in China ${ }^{(19)}$ reported that adherence to dietary patterns of refined grains (OR $=4 \cdot 9 ; 95 \%$ CI 1.4 , 17.0), high nuts, seeds, fat and soyabeans, low milk $(\mathrm{OR}=7 \cdot 5 ; 95 \% \mathrm{CI} 1 \cdot 8,32 \cdot 3)$, and sweets and seafood $(\mathrm{RR}=1.23 ; 95 \%$ CI $1.02,1.49)$ during pregnancy was associated with an increased likelihood of GDM.

\section{The association between dietary patterns and adverse birth outcomes (preterm birth and low birth weight)}

Dietary pattern and preterm birth

Based on a meta-analysis of nine studies ${ }^{(9,20,21,36,42-46)}$, women who had good adherence to a healthy dietary pattern were shown to have reduced odds of PTB $(\mathrm{OR}=$ 0.75 ; $95 \%$ CI $0.57,0.93)$, although significant heterogeneity was observed $\left(I^{2}=89 \cdot 6 \%, P=0 \cdot 0001\right.$; Fig. 4(a)). Further subgroup analysis indicated a difference in relation to dietary pattern assessment method (MDS, DDS or PCA; $P=0.001$ ). There was also a significant subgroup difference regarding dietary assessment period (second trimester or both second and third trimesters; $P=0 \cdot 001$; Fig. 4(b)).

On the other hand, the pooled estimate of four stu$\operatorname{dies}^{(9,21,42,43)}$ showed that a Western dietary pattern did not increase the odds of PTB (OR $=1 \cdot 11 ; 95 \%$ CI $0 \cdot 87$, $1.34 ; \quad I^{2}=77.8 \%, \quad P=0.004 ; \quad$ Fig. $\left.4(\mathrm{c})\right)$. There were 
(a) Study

Second and third trimesters
De Seymour et al. $(2016)^{(18)}$
Karamanos et al. $(2014)^{(40)}$
Subtotal $\left(I^{2}=85 \cdot 7 \%, P=0.008\right)$
Second trimester
He et al. $(2015)^{(10)}$
Nascimento et al. $(2016)^{(41)}$
Tryggvadottir et al. $(2016)^{(34)}$
Subtotal $\left(I^{2}=43.0 \%, P=0 \cdot 173\right)$
Overall $\left(I^{2}=68.6 \%, P=0.013\right)$

NOTE: Weights are from random-effects analysis

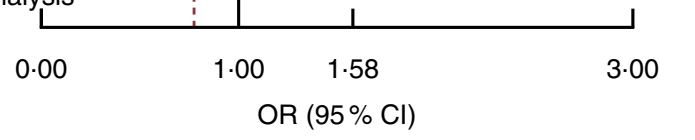

(b) Study Effect size $(95 \% \mathrm{Cl}) \quad(\%)$

\begin{tabular}{|c|c|c|}
\hline \multicolumn{3}{|l|}{ Second and third trimesters } \\
\hline Dayeon et al. (2015) & $22.30(3.90,127.40)$ & 0.00 \\
\hline De Seymour et al. (2016) $)^{(18)}$ & $0.96(0.79,1.17)$ & $46 \cdot 63$ \\
\hline Subtotal $\left(I^{2}=0.0 \%, P=0.498\right)$ & $0.96(0.77,1.15)$ & $46 \cdot 63$ \\
\hline \multicolumn{3}{|l|}{ Second trimester } \\
\hline $\mathrm{He}$ et al. (2015) $)^{(19)}$ & $0.95(0.78,1.16)$ & $46 \cdot 63$ \\
\hline Nascimento et al. (2016) $)^{(41)}$ & $0.78(0.43,1.43)$ & $6 \cdot 73$ \\
\hline Subtotal $\left(I^{2}=0.0 \%, P=0.533\right)$ & $0.93(0.75,1.11)$ & $53 \cdot 37$ \\
\hline \multirow{3}{*}{$\begin{array}{l}\text { Overall }\left(I^{2}=0.0 \%, P=0.825\right) \\
\text { NOTE: Weights are from random-effects } \\
\text { analysis }\end{array}$} & $0.94(0.81,1.07)$ & $100 \cdot 00$ \\
\hline & \multicolumn{2}{|c|}{-} \\
\hline & \multicolumn{2}{|c|}{$127 \cdot 00$} \\
\hline
\end{tabular}

Fig. 3 (colour online) Forest plot for the pooled OR of the association between gestational diabetes mellitus (GDM) and different dietary patterns, with subgroup analysis regarding period of dietary assessment (second trimester $v$. both second and third trimesters): (a) association between GDM and healthy dietary pattern; (b) association between GDM and Western dietary pattern. The study-specific OR and $95 \% \mathrm{Cl}$ are represented by the black diamond and the horizontal line, respectively; the area of the grey square is proportional to the specific-study weight to the overall meta-analysis. The centre of the blue open diamond and the red dashed vertical line represent the pooled OR; and the width of the blue open diamond represents the pooled $95 \% \mathrm{Cl}$

subgroup differences between assessing diet in the second trimester and in both the second and third trimesters with respect to risk of PTB $(P=0 \cdot 001)$. We did not undertake a subgroup analysis regarding study design, as all studies had the same design (cohort).

\section{Dietary patterns and low birth weight}

Two studies assessed the effect of dietary pattern during gestation on LBW. A study in Ghana ${ }^{(47)}$ reported that a 'health conscious' dietary pattern with a high intake of corn, rice, cassava, yam, fruits, vegetables (carrots, tomatoes, dark green leafy vegetables, cabbage, salad, cucumber), meat and eggs reduced the odds of LBW $(\mathrm{OR}=0.23 ; 95 \%$ CI $0.12,0.45)$. Similarly, that study reported that women who had a higher DDS were less likely to deliver an LBW baby $v$. those who had a lower DDS (OR $=0 \cdot 10 ; 95 \%$ CI 0.04, 0.13). However, a high consumption of sweetened beverages, ice cream, chocolate, energy drinks, milk and local soft drinks, which was labelled the 'non-health conscious' dietary pattern, was not significantly associated with LBW (OR $=1 \cdot 04 ; 95 \%$ CI $0.65,1.67)$. Another study in Ethiopia ${ }^{(46)}$ showed that women who had an adequate DDS were less likely to deliver an LBW baby (OR=0.49; 95\% CI 0.24, 0.97). 
(a)

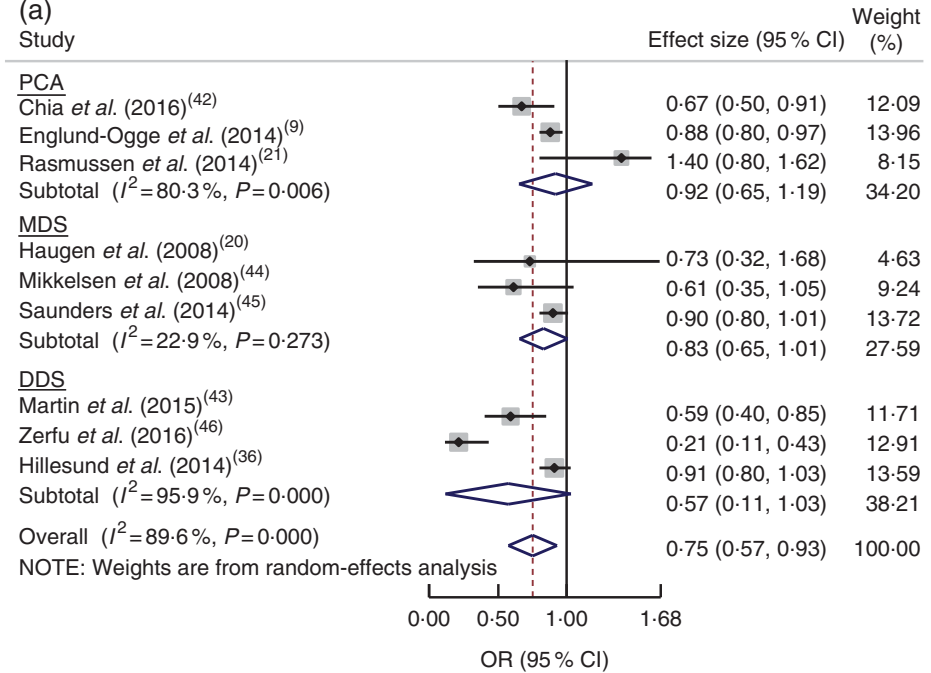

(b)

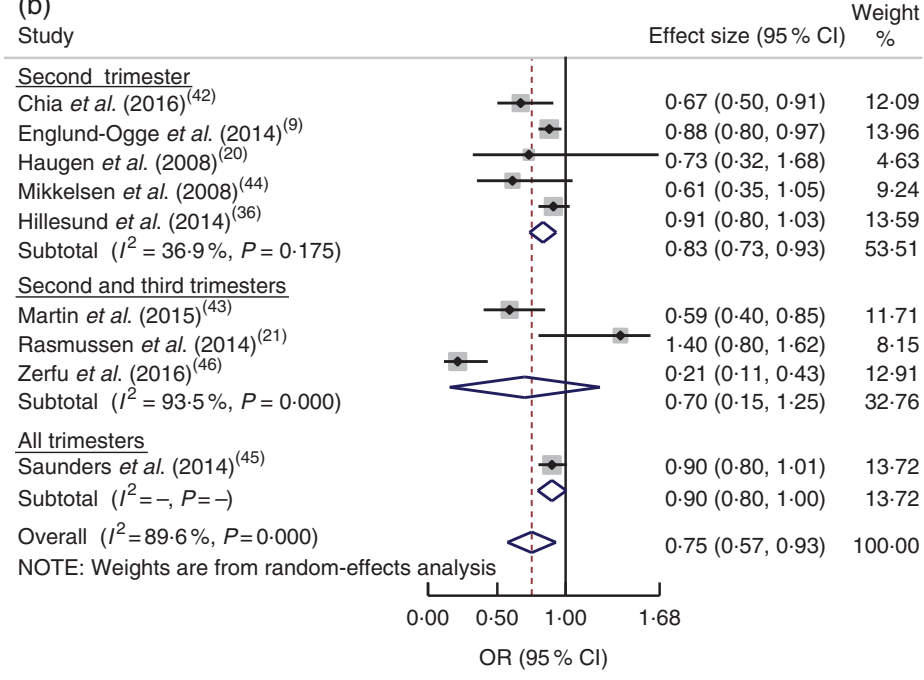

(c)

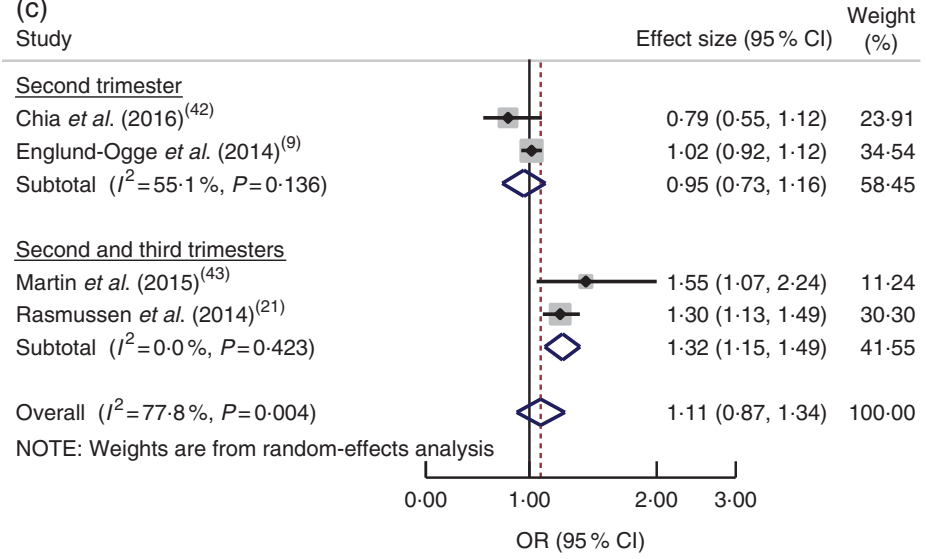

Fig. 4 (colour online) Forest plot for the pooled OR of the association between preterm birth (PTB) and different dietary patterns: (a) association between healthy dietary pattern and PTB, with subgroup analysis in relation to dietary pattern assessment methods (Mediterranean diet score (MDS) v. dietary diversity score (DDS) v. principal component analysis (PCA)); (b) association between healthy dietary pattern and PTB, with subgroup analysis regarding period of dietary assessment (second trimester $v$. both second and third trimesters $v$. all trimesters); and (c) association between Western pattern and PTB, with subgroup analysis regarding period of dietary assessment (second trimester $v$. both second and third trimesters). The study-specific OR and $95 \% \mathrm{Cl}$ are represented by the black diamond and the horizontal line, respectively; the area of the grey square is proportional to the specificstudy weight to the overall meta-analysis. The centre of the blue open diamond and the red dashed vertical line represent the pooled OR; and the width of the blue open diamond represents the pooled $95 \% \mathrm{Cl}$ 


\section{Discussion}

The current systematic review and meta-analysis summarizes evidence focusing on the effects of different dietary patterns during pregnancy on adverse pregnancy (HDP and GDM) and birth (PTB and LBW) outcomes. Globally, adverse pregnancy outcomes and nutritional insufficiencies still remain public health problems ${ }^{(48)}$. Sufficient consumption of energy, protein and micronutrients continues to be essential throughout pregnancy ${ }^{(49)}$.

\section{Hypertensive disorders of pregnancy}

The meta-analysis of four studies assessing the healthy diet pattern resulted in pooled estimates suggesting decreased odds of pre-eclampsia. However, other studies reported inconsistent findings on the association between adherence to a healthy dietary pattern and the likelihood of HDP occurrence. A cohort study in the Netherlands ${ }^{(25)}$ revealed that adherence to a Mediterranean diet pattern (vegetables, vegetable oils, pasta, fish, legumes and rice) or a traditional pattern (meat and potatoes) was not associated with gestational hypertension. A cohort study in Brazil ${ }^{(35)}$ revealed that adherence to healthy dietary patterns with high intakes of dairy products, fruit, green vegetables, legumes, fish, cakes, cookies-crackers and tea was not associated with a change in systolic or diastolic blood pressure. On the contrary, a cross-sectional study in Tanzania ${ }^{(37)}$ reported that, compared with a lower score, having a high and medium DDS were associated with increased odds of gestational hypertension.

These inconsistencies might be due to the differences in method and population characteristics. The Tanzanian study was cross-sectional ${ }^{(37)}$ and conducted in a resourcelimited setting; however, the other studies were cohort studies conducted in well-resourced settings, except the Brazilian study ${ }^{(35)}$. These studies also assessed dietary intake using a different number of food items and methods. The Tanzanian study applied a $24 \mathrm{~h}$ recall method using sixteen food groups, while the studies from Brazil ${ }^{(35)}$ and the Netherlands ${ }^{(25)}$ assessed dietary intake using an eightytwo-item and a 293-item FFQ, respectively.

The healthy diet pattern is in line with dietary guidelines, which suggest consumption of whole grains, vegetables, fruits, potatoes, pasta, cereals, beans, lentils and fish ${ }^{(50)}$. Similarly, the beneficiary influence of diets high in fibre, K, fruits, vegetables, cereals, dark bread and low-fat dairy products was reported as decreasing the odds of preeclampsia $^{(51)}$. It was also reported that a lower likelihood of pregnancy-induced hypertension or pre-eclampsia is observed with a diet comprising intake of plant-derived foodstuffs and vegetables ${ }^{(52)}$. The risk of pregnancy complications like pre-eclampsia and LBW has been linked with maternal oxidative stress in the middle of pregnancy ${ }^{(53)}$. Evidence indicates that oxidative stress during pregnancy could be reduced by antioxidant compounds from fruit and vegetables ${ }^{(54)}$. The findings of a multicentre study indicate that oxidative stress could be reduced by sufficient intakes of fruit, vegetables and vitamin $\mathrm{C}^{(54)}$. A combination of vitamin $\mathrm{C}$ and $\mathrm{E}$ might lower the risk of pre-eclampsia ${ }^{(55)}$ through removal of free radicals which may cause oxidative stress in pregnancy ${ }^{(56)}$. Therefore, it could be the cumulative effect of nutrients and their biochemical properties that influence pre-eclampsia risk.

\section{Gestational diabetes mellitus}

The meta-analyses of five studies assessing the healthy diet pattern resulted in pooled estimates that indicated reduced odds of GDM, but this was not statistically significant, most likely due to insufficient power, since few articles were included. Additionally, there were inconsistent findings among included studies for meta-analysis regarding the healthy dietary patterns and GDM; three studies showed decreased odds of GDM while the remainder reported no association. This might result from unmeasured factors due to the majority of studies not controlling for all possible confounding factors. For instance, He et $a l^{(19)}$ could not control for parity, energy intake, blood pressure and family history of type 2 diabetes mellitus. Likewise, parity, energy intake and blood pressure were not adjusted for in the other two studies ${ }^{(40,41)}$. There was also a difference in assessing dietary intake across these studies, with four studies ${ }^{(18,19,40,41)}$ using a validated FFQ and the other an unvalidated $\mathrm{FFQ}^{(34)}$. The dietary intake was assessed at different trimesters of pregnancy, even though there was no significant difference in subgroup analysis based on dietary intake assessment period. This could be a possible explanation for the variations across different studies.

Evidence indicates that pre-pregnancy adherence to a Mediterranean pattern style, with intake of fruit, vegetables, legumes, nuts, fish and cereals, and to the Dietary Approaches to Stop Hypertension (DASH) diet decreases the odds of $\mathrm{GDM}^{(57,58)}$. Similarly, a clinical trial reported that adhering to the DASH diet, which is high in fruits, vegetables, whole grains and low-fat dairy products, with low amounts of saturated fats, cholesterol and refined grains, reduced the need for insulin treatment ${ }^{(59)}$. Intake of fibre, fruits and cereals reduced the odds of $\mathrm{GDM}^{(60)}$.

A cohort study reported that higher odds of GDM was observed with adherence to a Western dietary pattern, which contained higher intake of refined-grain products, processed meat, red meat, French fries and pizza, sweets and desserts ${ }^{(26)}$. However, our pooled estimate of four studies did not show a significant relationship between the Western pattern and GDM occurrence. The possible explanation may be the difference in the dietary pattern investigation methods (two studies used $\mathrm{FFQ}^{(19,41)}$ and two studies used $24 \mathrm{~h}$ recall methods ${ }^{(18,39)}$ ) and population (one study was conducted in a Western population ${ }^{(39)}$ and three studies were done in an Asian population $\left.{ }^{(18,19,41)}\right)$. 


\section{Preterm birth}

In the current systematic review, a pooled estimate of nine studies indicated that compared with low adherence, higher adherence to a healthy dietary pattern significantly decreased the odds of PTB. Likewise, the pooled estimates of four studies on vegetable pattern and three studies on the Mediterranean diet indicated decreased odds of PTB, but this was not statistically significant. However, the meta-analysis result of four studies assessing the Western pattern and PTB showed that adherence to the Western pattern was not significantly associated with PTB. There were significant differences in subgroup analysis based on dietary intake assessment period. In two articles, the dietary intake was assessed in the second (13-27 weeks) and third (28-40 weeks) trimesters and reported that the Western dietary pattern significantly increased the odds of PTB. Nevertheless, the other two studies assessed the dietary intake in the second trimester (13-27 weeks) and the Western dietary pattern did not significantly increase the odds of PTB. A previous systematic review of randomized controlled trials revealed that macronutrient dietary interventions have reduced $\mathrm{PTB}^{(61)}$.

\section{Low birth weight}

Two articles assessed the effect of dietary patterns on LBW. A dietary pattern labelled as 'health conscious', characterized by intake of local dishes made from corn flour, vegetables (carrot, tomatoes, dark green leafy vegetables, cabbage, salad, cucumber), rice, meat, a mixture of corn and cassava dough, yam, fruits, water and eggs, was associated with reduced odds of $\mathrm{LBW}^{(47)}$. Similarly, women who had a higher DDS were less likely to deliver an LBW baby ${ }^{(46,47)}$. However, high consumption of sweetened beverages, ice cream, chocolate, energy drinks, milk and local soft drinks, which was labelled as a 'non-health conscious' dietary pattern, showed a significant effect on risk of $\mathrm{LBW}^{(47)}$. This is in line with the evidence that suggests the occurrence of LBW has decreased through the consumption of foods and fortified foodstuffs ${ }^{(62)}$.

It is suggested that pregnant women should be advised to eat a diet rich in fruits and vegetables, whole grains, beans, lean meats and fish/seafood, and low in added sugar, red meat and processed foods ${ }^{(63)}$. Intake of vegetables, fruits and legumes improves micronutrient and antioxidant intakes, which could improve pregnancy and birth outcomes ${ }^{(63)}$, particularly at the second trimester since oxidative stress has been shown to reach high levels mid-pregnancy ${ }^{(64)}$. Pregnancy complications and adverse outcomes like preeclampsia and PTB have been related to oxidative stress and associated inflammation ${ }^{(53)}$. Antioxidant vitamins ( $\mathrm{C}$ and E) and essential trace elements ( $\mathrm{Cu}$ and $\mathrm{Zn}$ ) through dietary intake of legumes and fruits, which are rich in these nutrients, could decrease this risk ${ }^{(65-67)}$. Oxidative stress-linked adverse pregnancy outcomes could be reduced by antioxidants through an intake of vegetables and fruits ${ }^{(68)}$.

\section{Study limitations}

The limitations of the present systematic review must be acknowledged. To acquire complete dietary data, most of the articles reviewed applied FFQ followed by diet scores. Nevertheless, there are unavoidable dietary intake misclassifications, which probably bias the degree of detecting real effects. Furthermore, problems of recall bias are also unavoidable because dietary information is dependent on memory. Including articles written only in the English language is another shortcoming of the systematic review. Due to the nature of nutritional research, it is difficult to make all dietary exposures similar for all study subjects. Heterogeneity among studies is a further issue; however, meta-analysis permits the inconsistent findings among studies to be evaluated, even with heterogeneity ${ }^{(69)}$. As all included studies were observational epidemiological studies, the effect of confounders may be another limitation of the current review, despite controlling for some possible confounding factors. Publication bias is always a concern in any review. Reviewed studies that had negative results might not have been submitted for publication, and thus are less likely to have been published.

\section{Conclusion}

The evidence presented in the current systematic review indicates the inconsistent associations between different dietary patterns and pregnancy and birth outcomes. Some results in the systemic review show the importance of healthy dietary intake during gestation to improve pregnancy and birth outcomes for the mother and infant, even though inconsistencies have been observed among studies. Essentially, the review suggests that dietary patterns with higher intake of whole grains, vegetables/fruits, legumes and fish are associated with lower likelihood of adverse pregnancy and birth outcomes. However, as the evidence presented herein is inconsistent regarding the association between dietary intake and pregnancy and birth outcomes, caution should be given during advising pregnant women about diet. Since most of the articles included in the review were conducted in resource-rich settings, additional studies need to be done in resource-limited settings to elucidate the impact of limited resources on dietary intake and adverse pregnancy and birth outcomes.

\section{Acknowledgements}

Acknowledgements: The authors would like to thank Debbie Booth for her help with developing the literature search strategy and use of Endnote. The authors would also like to thank Dr Ryan O'Neill for his unreserved help in editing the English language. Financial support: This research received no specific grant from any funding agency in the public, commercial or not-for-profit sectors. 
Conflict of interest: The authors declare that they have no conflicting interests. Authorship: K.T.K., C.C., D.L. and E.G. formulated the research questions. K.T.K., C.C., D.L. and E.G. designed the study. K.T.K., C.C., D.L., E.G. and T.K.T. carried out the analysis. K.T.K., C.C., D.L., E.G. and T.K.T. analysed the data and wrote the manuscript. Ethics of buman subject participation: Not applicable.

\section{References}

1. American College of Obstetricians and Gynecologists (2013) Hypertension in pregnancy. Report of the American College of Obstetricians and Gynecologists' Task Force on Hypertension in Pregnancy. Obst Gynecol 122, 1122-1131.

2. Say L, Chou D, Gemmill A et al. (2014) Global causes of maternal death: a WHO systematic analysis. Lancet Glob Health 2, e323-e333.

3. Gemmell L, Martin L, Murphy KE et al. (2016) Hypertensive disorders of pregnancy and outcomes of preterm infants of 24 to 28 weeks' gestation. J Perinatol 36, 1067-1072.

4. Lawn JE, Gravett MG, Nunes TM et al. (2010) Global report on preterm birth and stillbirth (1 of 7): definitions, description of the burden and opportunities to improve data. BMC Pregnancy Childbirth 10, Suppl. 1, S1.

5. Blencowe H, Cousens S, Chou D et al. (2013) Born too soon: the global epidemiology of 15 million preterm births. Reprod Health 10, Suppl. 1, S2.

6. Blencowe H, Cousens S, Oestergaard MZ et al. (2012) National, regional, and worldwide estimates of preterm birth rates in the year 2010 with time trends since 1990 for selected countries: a systematic analysis and implications. Lancet 379, 2162-2172.

7. Ramakrishnan U (2004) Nutrition and low birth weight: from research to practice. Am J Clin Nutr, 79, 17-21.

8. Mahumud RA, Sultana M \& Sarker AR (2017) Distribution and determinants of low birth weight in developing countries. J Prev Med Public Health 50, 18-28.

9. Englund-Ogge L, Brantsaeter AL, Sengpiel V et al. (2014) Maternal dietary patterns and preterm delivery: results from large prospective cohort study. BMJ 348, g1446.

10. Brantsaeter AL, Haugen M, Samuelsen SO et al. (2009) A dietary pattern characterized by high intake of vegetables, fruits, and vegetable oils is associated with reduced risk of preeclampsia in nulliparous pregnant Norwegian women. $J$ Nutr 139, 1162-1168.

11. Jacques PF \& Tucker KL (2001) Are dietary patterns useful for understanding the role of diet in chronic disease? Am J Clin Nutr 73, 1-2.

12. Hu FB (2002) Dietary pattern analysis: a new direction in nutritional epidemiology. Curr Opin Lipidol 13, 3-9.

13. Wirfält E, Drake I \& Wallström P (2013) What do review papers conclude about food and dietary patterns? Food Nutr Res 57, 20523.

14. Kant AK (2004) Dietary patterns and health outcomes. J Am Diet Assoc 104, 615-635.

15. Food Agriculture and Organization of the United Nations \& Family Health International 360 (2016) Minimum Dietary Diversity for Women: A Guide for Measurement. Rome: FAO.

16. Ocke MC (2013) Evaluation of methodologies for assessing the overall diet: dietary quality scores and dietary pattern analysis. Proc Nutr Soc 72, 191-199.

17. Rifas-Shiman SL, Rich-Edwards JW, Kleinman KP et al. (2009) Dietary quality during pregnancy varies by maternal characteristics in Project Viva: a US cohort. J Am Diet Assoc 109, 1004-1011.
18. De Seymour J, Chia A, Colega M et al. (2016) Maternal dietary patterns and gestational diabetes mellitus in a multiethnic Asian cohort: the GUSTO study. Nutrients 8, E574.

19. He JR, Yuan MY, Chen NN et al. (2015) Maternal dietary patterns and gestational diabetes mellitus: a large prospective cohort study in China. Br J Nutr 113, 1292-1300.

20. Haugen M, Meltzer HM, Brantsaeter AL et al. (2008) Mediterranean-type diet and risk of preterm birth among women in the Norwegian Mother and Child Cohort Study (MoBa): a prospective cohort study. Acta Obstet Gynecol Scand 87, 319-324.

21. Rasmussen MA, Maslova E, Halldorsson TI et al. (2014) Characterization of dietary patterns in the Danish national birth cohort in relation to preterm birth. PLoS One 9, e93644.

22. Kiøllesdal MKR \& Holmboe-Ottesen G (2014) Dietary patterns and birth weight - a review. AIMS Public Health 1, 211-225.

23. Agrawal S, Fledderjohann J, Vellakkal S et al. (2015) Adequately diversified dietary intake and iron and folic acid supplementation during pregnancy is associated with reduced occurrence of symptoms suggestive of pre-eclampsia or eclampsia in Indian women. PLoS One 10, e0119120.

24. Borgen I, Aamodt G, Harsem N et al. (2012) Maternal sugar consumption and risk of preeclampsia in nulliparous Norwegian women. Eur J Clin Nutr 66, 920-925.

25. Timmermans S, Steegers-Theunissen RPM, Vujkovic M et al. (2011) Major dietary patterns and blood pressure patterns during pregnancy: the Generation R Study. Am J Obstet Gynecol 205, 337.e1-e12.

26. Zhang C, Schulze MB, Solomon CG et al. (2006) A prospective study of dietary patterns, meat intake and the risk of gestational diabetes mellitus. Diabetologia 49, 2604-2613.

27. Poon AK, Yeung E, Boghossian N et al. (2013) Maternal dietary patterns during third trimester in association with birthweight characteristics and early infant growth. Scientifica (Cairo) 2013, 786409.

28. Academy of Nutrition and Dietetics (2016) Evidence Analysis Manual: Steps in the Academy Evidence Analysis Process. Chicago, IL: Research, International and Strategic Business Development Team, Academy of Nutrition and Dietetics; available at https://www.andeal.org/vault/2440/ web/files/2016_April_EA_Manual.pdf

29. Zhang J \& Yu KF (1998) What's the relative risk? A method of correcting the odds ratio in cohort studies of common outcomes. JAMA 280, 1690-1691.

30. Higgins JP \& Green S (editors) (2011) Cochrane Handbook for Systematic Reviews of Interventions Version 5.1.0. The Cochrane Collaboration; available at http://www.handbook.cochrane.org

31. Wiest MM, Lee KJ \& Carlin JB (2015) Statistics for clinicians: An introduction to logistic regression. I Paediatr Child Health 51, 670-673.

32. Pryor ER (2010) Logistic Regression: A Self-Learning Text, 3rd ed. New York: Springer.

33. Borenstein M, Hedges LV, Higgins JPT et al. (2009) Introduction to Meta-Analysis, 1st ed. Chichester: John Wiley \& Sons Ltd.

34. Tryggvadottir EA, Medek H, Birgisdottir BE et al. (2016) Association between healthy maternal dietary pattern and risk for gestational diabetes mellitus. Eur J Clin Nutr 70, 237-242.

35. Eshriqui I, Vilela AAF, Rebelo F et al. (2016) Gestational dietary patterns are not associated with blood pressure changes during pregnancy and early postpartum in a Brazilian prospective cohort. Eur J Nutr 55, 21-32.

36. Hillesund ER, Overby NC, Engel SM et al. (2014) Associations of adherence to the New Nordic Diet with risk of preeclampsia and preterm delivery in the Norwegian Mother and Child Cohort Study (MoBa). Eur J Epidemiol 29, $753-765$. 
37. Mwanri AW, Kinabo JL, Ramaiya K et al. (2015) High blood pressure and associated risk factors among women attending antenatal clinics in Tanzania.J Hypertens 33, 940-947.

38. Torjusen H, Brantsaeter AL, Haugen M et al. (2014) Reduced risk of pre-eclampsia with organic vegetable consumption: results from the prospective Norwegian Mother and Child Cohort Study. BMJ Open 4, e006143.

39. Dayeon S, Kyung Won L \& Won OS (2015) Dietary patterns during pregnancy are associated with risk of gestational diabetes mellitus. Nutrients 7, 9369-9382.

40. Karamanos B, Thanopoulou A, Anastasiou E et al. (2014) Relation of the Mediterranean diet with the incidence of gestational diabetes. Eur J Clin Nutr 68, 8-13.

41. Nascimento GR, Alves LV, Fonseca CL et al. (2016) Dietary patterns and gestational diabetes mellitus in a low income pregnant women population in Brazil - a cohort study. Arch Latinoam Nutr 66, 301-308.

42. Chia AR, de Seymour JV, Colega M et al. (2016) A vegetable, fruit, and white rice dietary pattern during pregnancy is associated with a lower risk of preterm birth and larger birth size in a multiethnic Asian cohort: the Growing Up in Singapore Towards healthy Outcomes (GUSTO) cohort study. Am J Clin Nutr 104, 1416-1423.

43. Martin CL, Sotres-Alvarez D \& Siega-Riz AM (2015) Maternal dietary patterns during the second trimester are associated with preterm birth. J Nutr 145, 1857-1864.

44. Mikkelsen TB, Osterdal ML, Knudsen VK et al. (2008) Association between a Mediterranean-type diet and risk of preterm birth among Danish women: a prospective cohort study. Acta Obstet Gynecol Scand 87, 325-330.

45. Saunders L, Guldner L, Costet N et al. (2014) Effect of a Mediterranean diet during pregnancy on fetal growth and preterm delivery: results from a French Caribbean MotherChild Cohort Study (TIMOUN). Paediatr Perinat Epidemiol 28, 235-244.

46. Zerfu TA, Umeta M \& Baye K (2016) Dietary diversity during pregnancy is associated with reduced risk of maternal anemia, preterm delivery, and low birth weight in a prospective cohort study in rural Ethiopia. Am J Clin Nutr 103, 1482-1488.

47. Abubakari A \& Jahn A (2016) Maternal dietary patterns and practices and birth weight in northern Ghana. PLoS One 11, $\mathrm{e} 0162285$.

48. Gernand AD, Schulze KJ, Stewart CP et al. (2016) Micronutrient deficiencies in pregnancy worldwide: health effects and prevention. Nat Rev Endocrinol 12, 274-289.

49. Wakimoto P, Akabike A \& King JC (2015) Maternal nutrition and pregnancy outcome - a look back. Nutr Today 50, 221-229.

50. World Health Organization (2001) Healthy Eating during Pregnancy and Breastfeeding: Booklet for Mothers. Copenhagen: WHO Regional Office for Europe, Nutrition and Food Security.

51. Frederick IO, Williams MA, Dashow E et al. (2005) Dietary fiber, potassium, magnesium, and calcium in relation to preeclampsia risk. J Reprod Med 50, 322-344.

52. Pistollato F, Sumalla Cano S, Elio I et al. (2015) Plant-based and plant-rich diet patterns during gestation: beneficial effects and possible shortcomings. Adv Nutr 6, 581-591.
53. Hsieh TT, Chen SF, Lo LM et al. (2012) The association between maternal oxidative stress at mid-gestation and subsequent pregnancy complications. Reprod Sci 19, 505-512.

54. Kim H, Hwang JY, Ha EH et al. (2011) Fruit and vegetable intake influences the association between exposure to polycyclic aromatic hydrocarbons and a marker of oxidative stress in pregnant women. Eur J Clin Nutr $\mathbf{6 5}$, 1118-1125.

55. Raijmakers MT, Dechend R \& Poston L (2004) Oxidative stress and preeclampsia: rationale for antioxidant clinical trials. Hypertension 44, 374-380.

56. Siddiqui IA, Jaleel A, Tamimi W et al. (2010) Role of oxidative stress in the pathogenesis of preeclampsia. Arch Gynecol Obstet 282, 469-474.

57. Tobias DK, Cuilin Z, Chavarro J et al. (2012) Prepregnancy adherence to dietary patterns and lower risk of gestational diabetes mellitus. Am J Clin Nutr 96, 289-295.

58. Schoenaker D, Soedamah-Muthu SS \& Mishra GD (2016) Quantifying the mediating effect of body mass index on the relation between a Mediterranean diet and development of maternal pregnancy complications: the Australian Longitudinal Study on Women's Health. Am J Clin Nutr 104, 638-645.

59. Asemi Z, Samimi M, Tabassi Z et al. (2014) The effect of DASH diet on pregnancy outcomes in gestational diabetes: a randomized controlled clinical trial. Eur J Clin Nutr 68, 490-495.

60. Zhang C, Liu S, Solomon CG et al. (2006) Dietary fiber intake, dietary glycemic load, and the risk for gestational diabetes mellitus. Diabetes Care 29, 2223-2230.

61. Gresham E, Bisquera A, Byles JE et al. (2016) Effects of dietary interventions on pregnancy outcomes: a systematic review and meta-analysis. Matern Child Nutr 12, 5-23.

62. Gresham E, Byles JE, Bisquera A et al. (2014) Effects of dietary interventions on neonatal and infant outcomes: a systematic review and meta-analysis. Am J Clin Nutr 100, $1298-1321$.

63. King JC (2006) Maternal obesity, metabolism, and pregnancy outcomes. Annu Rev Nutr 26, 271-291.

64. Casanueva E \& Viteri FE (2003) Iron and oxidative stress in pregnancy. J Nutr 133, 5 Suppl. 2, 1700S-1708S.

65. Mistry HD \& Williams PJ (2011) The importance of antioxidant micronutrients in pregnancy. Oxid Med Cell Longev 2011, 841749 .

66. Al-Gubory KH, Fowler PA \& Garrel C (2010) The roles of cellular reactive oxygen species, oxidative stress and antioxidants in pregnancy outcomes. Int J Biochem Cell Biol 42, $1634-1650$.

67. Zhang Y, Zhou H, Perkins A et al. (2017) Maternal dietary nutrient intake and its association with preterm birth: a case-control study in Beijing, China. Nutrients 9, E221.

68. Asemi Z, Samimi M, Tabassi Z et al. (2013) A randomized controlled clinical trial investigating the effect of DASH diet on insulin resistance, inflammation, and oxidative stress in gestational diabetes. Nutrition 29, 619-624.

69. Higgins JP, Thompson SG, Deeks JJ et al. (2003) Measuring inconsistency in meta-analyses BMJ 327, 557-560. 